Ribeiro, F.S., and Costa, D.B. (2018). "Last Planner System: Implementation and Evaluation with Focus on the Phase Schedule." In: Proc. $26^{\text {th }}$ Annual Conference of the International. Group for Lean Construction (IGLC), González, V.A. (ed.), Chennai, India, pp. 702-712. DOI: doi.org/10.24928/2018/0411. Available at: www.iglc.net.

\title{
LAST PLANNER SYSTEM: IMPLEMENTATION AND EVALUATION WITH FOCUS ON THE PHASE SCHEDULE
}

\author{
Flora S. Ribeiro', and Dayana B. Costa ${ }^{2}$
}

\begin{abstract}
Phase Schedule is a Last Planner System practice whose role, both from a practical and theoretical point of view, is still being debated in the construction industry. Thus, there is a need for a better understanding of Phase Schedule implementation practices and the impacts of those on production planning and control. This paper presents the results of the implementation and evaluation of the LPS focusing on the Phase Schedule practices based on two in-depth case studies developed from April 2016 to August 2017 in Salvador-Brazil.

The Case Studies involved the implementation of the LPS and the Phase Schedule practices and the analysis of the impact of using those practices on the production planning and control processes. The findings indicated that the cycles of the Phase Schedule improve the constraints analysis, collaboration between those involved, transparency in the planning process, adherence between levels planning by using performance metrics, reliability of plans and commitment to the deadlines. Also, the activities which were initially not analyzed as critical, have strong influence on the performance of the production planning and control.
\end{abstract}

\section{KEYWORDS}

Last Planner System, Phase Schedule, Collaboration.

\section{INTRODUCTION}

The development of the Last Planner System (LPS) established several changes in the way construction projects were planned and controlled. According to Ballard (2000), Last Planner can be understood as a mechanism for transforming what SHOULD be done into what CAN be done, forming an inventory of ready work, from which the Weekly Work Plan (WWP)can be formed. The LPS is divided into different planning levels (Ballard andTommelein, 2016):master scheduling, which the milestones and phases durations

Civil Engineer, Master Student, Federal University of Bahia, floraseixasribeiro@gmail.com

Associate Professor, Graduate Program in Civil Engineering, Federal University of Bahia, dayanabcosta@ufba.br 
and overlaps are set; phase scheduling, where the team specify the handoffs and conditions of satisfaction between processes within phases; look ahead planning, where the constraints are identified and removed, the tasks breakdown from processes into operationsoccurs and the operations are designed; commitment planning, during which reliable promises are made; and learning, where plan failures ae analyzed in search of countermeasures.

Since its development, several studies have been conducted to analyze and develop LPS. Daniel et al. (2015) studied the components of the LPS implemented and found that the measuring of Percentage Plan Completed, Weekly Work Planning meeting, and recording reasons for non-completion are the commonly implemented components. Despite this LPS use, Hamzeh et al. (2012) state that in many construction projects, the implementation of the lookahead planning is deficient, resulting in a gap between master scheduling and commitment planning. While lookahead planning is built on master scheduling, a connection is rarely maintained between these two (Choo and Tommelein, 2000).Thus, there is a lack of adherence between planning levels.

Emdanat and Azambuja (2016) affirm that the alignment of commitment planning and master scheduling requires a systematic adherence to the processes of the LPS workflow from Phase Schedule to Weekly Work Planning and Commitment Management, and, the continuous capture of the data in an integrated and uniform way. In addition, Ballard and Tommelein (2016) state that there is a lack of studies which analyze a methodology that standardizes the adherence between the levels of Phase Schedule, lookahead and commitment planning. There is also a need to develop metrics to analyze the LPS performance focusing on the Phase Schedule's plan quality and reliability and measures to avoid plan failures (Ballard and Tommelein, 2016).

Based on the literature review, the research questions raised were: "How to implement the LPS focusing on the Phase Schedule, aiming to integrate the hierarchical planning levels?" And "How to evaluate the LPS performance, focusing on the Phase Schedule?". Therefore, the main objective of this research is to implement and evaluate the LPS, focusing on the Phase Schedule practices. Two in-depth case studies were carried out in Salvador-Brazil to achieve this objective.

\section{PHASE SCHEDULE AND METRICS TO SUPPORT THE ADHERENCE BETWEEN PLANNING LEVELS}

The Phase Schedule is one level in LPS, where a phase gets broken out from the master plan, in which milestones define phases, and people responsible for the work in that phase jointly develop the plan (Ballard and Tommelein, 2016). The practices of the Phase Schedule involve the technique of apull planning, whichis used to develop a plan for doing work at any level of task breakdown (Ballard and Tommelein, 2016). Also, it uses post-its and mural highlighting for the weeks that will be planned. Some LPS studies discuss the Phase Schedule, such as Knapp et al. (2006) and Kalsaas et al. (2009), which indicated that the teams better understood their project, their individual roles and what was required for the success of the project and analyzed their constraints in advance. 
More recently, studies related to the analysis of metrics that can support the adherence between plan levels in the LPS were presented. Emdanat and Azambuja (2016) analyzed several data from LPS implementations and identified metrics that allow the integration between planning levels. A set of the metrics identified in the literature may support the adherence and integration between planning levels, based on Ribeiro (2018), such as (Figure 1):

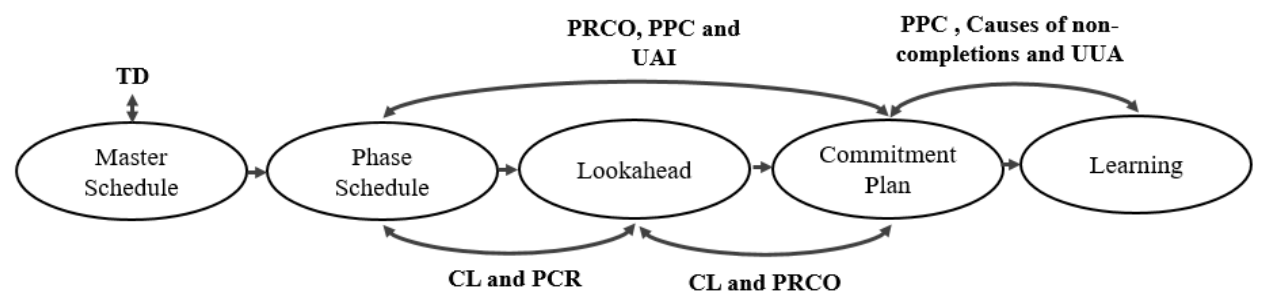

Figure 1: Indicators and interactions between the LPS planning levels

- Time Deviation (TD):evaluates the performance of the work, through the relation between the expected deadline and the effective deadline (Costa et al. 2005);

- Commitment Level (CL):evaluates the level of commitment of participants in Phase Schedule meetings to perform planned activities within the defined deadline (Emdanat and Azambuja, 2016). It is measured by the percentage of the ratio between the activities ready to be executed (constraints removed) and the total number of activities planned during the Phase Schedule cycles;

- Percentage of Constraint Removal (PCR):evaluates the effectiveness of lookahead planning in relation to the process of removing constraints (Jang and Kim, 2007);

- Percent Required Completed or Ongoing (PRCO):evaluates how many of the activities that were ready to be executed were actually completed or are certain to be finalized within the expected deadline (Emdanat and Azambuja, 2016);

- Percent Planned Complete (PPC): is a number of planned activities completed, divided by the total number of planned activities, and expressed as a percentage (Ballard, 2000); and Cause of non-completion plan: investigation of the causes for non-completion of the plans (Ballard, 2000).

- Unplanned Activities Included in the commitment planning (UAI):evaluates the percentage of activities included in the commitment planning that were not planned in Phase Schedule(Ribeiro, 2018).

- Unplanned and Unfinished Activities in the commitment planning (UUA): evaluates the percentage of activities in the commitment planning unfinished and not planned in Phase Schedule(Ribeiro, 2018).

\section{RESEARCH METHOD}

This research was divided into four phases: 1) literature review to find the knowledge gaps; 2) development of the Case Study A to implement the LPS and the Phase Schedule practices; 3) development of the Case Study B to incorporate the LPS and the Phase 
Schedule practices; and 4) development of a set of guidelines to implement and evaluate the LPS focusing on the Phase Schedule practices and identification, as well as the identification of the theoretical contribution of the study.

\section{CASE STUDY A AND B}

The case study A was developed during the construction of a clinical facility, from April to December 2016, located in the city of Salvador, Bahia, Brazil. The project team had already used elements of the LPS, such as visual controls, daily huddles and PPC metric. However, it was their first experience using the Phase Schedule practices.

The implementation of LPS focusing on the Phase Schedule practices occurred over 33 weeks, which included the Phase Schedule Cycle. The Phase Schedule cycle occurred through the following steps: (a) preparation, that occurred through training sessions and the phase definition; (b) meetings, which analysed the activities of the phase by using techniques (reverse plan, post it and board); and (c) monitoringof the activities and constraints planned, through performance measures and the evaluation of the implementation. A total of five cycles of Phase Schedule implementation was developed for the phases of foundation, structure, masonry, electrical and plumbing installation, facade, ceiling plaster board and drywall system. These meetings were attended by the project engineering team, subcontractors and the research team. Between these construction phases, weekly-basis meetings were heldto monitor the LPS implementation. The Phase Schedule meetings and the monitoring visits occurred at the construction site and lasted for an average of two hours.

The case study B was developed during the construction of a commercial building, from December 2016 to August 2017, also located in the city of Salvador, Bahia, Brazil. The project team already had experience with the Phase Schedule practices from the Study A.

The implementation of the LPS and the Phase Schedule cycles occurred in a similar way to the Study A, over 39 weeks. The meetings were also attended by the project engineering team, subcontractors and the research team. A total of seven Phase Schedule cycle was developed during this period, involving the following construction phases: structure, masonry, electrical and plumbing installation, internal mortar plaster, levelling mortar, facade, drywall system and internal painting. Every two weeks, meetings, with a total of 20 meetings, were held to monitor the LPS implementation during this period. The meetings also occurred at the construction site and lasted for an average of one hour. This reduced time was justified by the learning effect of those involved.

In addition, data from document analysis and interviews were collected during each phase of the case studies (Table 1).

Table 1: Stages of the Phase Schedule implementation cycle - Case Studies A and B

\begin{tabular}{|l|l|l|l|}
\hline $\begin{array}{c}\text { Stages of the } \\
\text { implementation cycle }\end{array}$ & \multicolumn{1}{|c|}{ Techniques or tools } & $\begin{array}{c}\text { People involved in the } \\
\text { Case Study A }\end{array}$ & $\begin{array}{c}\text { People involved in } \\
\text { the Case Study B }\end{array}$ \\
\hline \multirow{3}{*}{$\begin{array}{l}\text { Phase Schedule } \\
\text { preparation }\end{array}$} & Construction projects & 1 Production Engineer & 1 Production \\
& Master planning in the & 2 interns & Engineer \\
& MSProject tool & 5 foremen & 3 interns \\
\cline { 2 - 4 } & Excel sheets & foreman and 3 \\
\hline
\end{tabular}




\begin{tabular}{|l|l|l|l|}
\hline $\begin{array}{c}\text { Stages of the } \\
\text { implementation cycle }\end{array}$ & \multicolumn{1}{|c|}{ Techniques or tools } & $\begin{array}{l}\text { People involved in the } \\
\text { Case Study A }\end{array}$ & $\begin{array}{l}\text { People involved in } \\
\text { the Case Study B }\end{array}$ \\
\hline $\begin{array}{l}\text { Phase Schedule } \\
\text { meetings }\end{array}$ & $\begin{array}{l}\text { Board and Post its } \\
\text { Construction projects } \\
\text { Excel sheets }\end{array}$ & subcontractors & $\begin{array}{l}\text { supervisors } \\
5 \text { supervisors of the } \\
\text { subcontractors }\end{array}$ \\
\cline { 1 - 2 } $\begin{array}{l}\text { Monitoring of the } \\
\text { planned activities and } \\
\text { constraints }\end{array}$ & $\begin{array}{l}\text { Excel sheets for the } \\
\text { monitoring of what was } \\
\text { planned in the meetings }\end{array}$ & & \\
\hline Process evaluation & Structured Questionnaire & & \\
\hline
\end{tabular}

\section{DATA ANALYSIS}

Based on the literature review and the Case Studies A and B, it was possible to understand that the (a) effectiveness of the Phase Schedule practices, which are the techniques used (reverse planning, board, post-it) are concerned to collaboration and transparency and (b) the effectiveness of the processes of the LPS, which are the procedures the team will perform to achieve the defined goals (monitoring of the activities and constraints, use of metrics, etc), are related to adherence between planning levels, reliability of plans and commitment to the deadlines. The metrics selected to evaluate the LPS and the Phase Schedule were presented in the literature review and Figure 1. Thus a set of constructs and variables were proposed for data analysis, defined as follows:

- Collaboration: this is related to the team's ability to make joint decisions, based on shared knowledge among those involved, encouraging their commitment to the execution of activities. The variables are:(a) commitment to the execution of activities; (b) joint decision making; and (c) shared knowledge.The main sources of evidence used were participant observation, data analysis and interviews.

- Transparency: this is associated with the visualization of the necessary information in a simplified way and improved communication among those involved in the Phase Schedule. The variables are: (a) visual tools; (b) simplification of the information; quick understanding; (c) better communication among those involved; (d) easy visualization of the phase attack plan; (e) and verification of activities that would be performed simultaneously. Participant observation, data analysis and interviews were the main sources of evidence.

- Adherence between Planning Levels: this is associated with the uninterrupted flow of information between different planning levels, which can support the decision making at different planning levels. The variablesare: (a) conducting Phase Schedule practices; (b) analysis of master schedule goals; and (c) monitoring of the planned activities at different planning levels - joint analysis of the selected indicators. Participant observation, data analysis, performance metrics and interviews were the main sources of evidence.

- Reliability of Plans: aims to examine whether the phase was planned in a real way and was possible to execute as planned. The variables are: (a) execution of activities as planned - analysis of selected metrics; (b) monitoring of what was planned by the team involved in the Phase Schedule meetings; and (c) 
commitment to the constraints removal - analysis of selected metrics. Participant observation, data analysis, performance metrics and interviews were the sources of evidence.

- Commitment to the Deadlines: aims to verify whether the planned activities have been completed within the established deadline and in accordance with the master schedule, from which the goals were defined. Thevariables are: (a) execution of the activities in the duration planned; and (b) activities completed on the master schedule dates. Participant observation, data analysis, performance metrics and interviews were the main sources of evidence.

\section{RESULTS AND DISCUSSION}

\section{CASE STUDY A}

Based on the five Phase Schedule cycles carried out with the team involved and the 33 weeks of data collection, a set of lessons learned were identified concerning the factors that promote a more collaborative environment. A good practice identified was the earlier discussions for setting goals among the production engineer and the person in charge for the construction activity in the phase analyzed. Identified during the phase schedule meeting was that the reverse plan methodology and visual tools (post it and mural) support the joint definition of the best way to perform the activities, as well as the verification of possible interferences and constraints of activities. This result was confirmed by the interviewees who stated that the visualization of the project as whole, through the visual tools, simplified the information and made the activities easy to understand. During the execution of the activities, a self-reorganization and better interaction between the team involved in the Phase Schedule cycles when there were changes in the plan were observed as an important achievement.

By the joint analysis of the metrics selected, the analysis of adherence between planning levels were carried out. Figure 2 presents the results of the indicators CL and PCR and the indicators PRCO and PPC. The control cycle adopted for the analysis was 30 days, therefore more than one control cycle could be observed in some of the phases. The phases analyzed were Foundation (FO), Structure (S), Masonry and Electrical and Plumbing Installation (M/EP), Facade (FA), Ceiling Plaster Board and Drywall System (CPB/DS). 

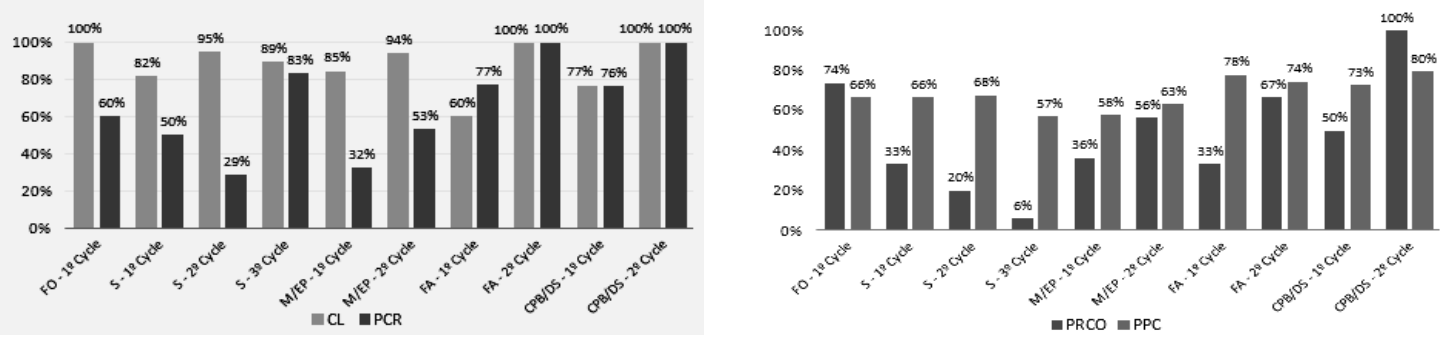

Figure 2: Indicators CL and PCR and PRCO and PPC results of the Case Study A

The results of PCR indicator (average of 66\%) indicated that many constraints were not removed until the due date established in the Phase Schedule/lookahead planning; however, there was a greater effort by the team to remove the constraints at the last moment (after the due date but before the start date of the activities). This can be seen through the results of the CL indicator (average of 88\%), which show the level of commitment of participants in Phase Schedule meetings to perform planned activities within the defined deadline (first commitment), thus improving the reliability of the plans. However, the second commitment, which is to finish the activities on the dates planned, was not attained by the team, evident in the PRCO results (average of $48 \%$ ). As it was the first time that the team had to define the execution's final dates with a greater anticipation of the execution date, this low PRCO result occurred due to the lackof experience to define the execution's final dates, compromising the deadlines established in the Phase Schedule cycles.

The PPC involved two types of activities: the ones planned in the phase schedule and the ones not planned. The activities not analyzed in the Phase Schedule/lookahead planning had an impact on the PPC average (68\%), as can be observed by the UAI indicator results, where $63 \%$ of the activities included in the commitment planning were related to those activities. Corroborating this, the UUA indicator results showed that an average of $58 \%$ of the uncompleted activities in the commitment planning were related to the activities not planned in the Phase Schedule/lookahead planning. Also, the late hiring of the electrical and plumbing subcontractor caused the delay in accomplishing the deadlines established in the master schedule, evaluated by the TD metric.

\section{CASE STUDY B}

As in the Case Study A, a set of lessons were learned based on the seven Phase Schedule cycles and the 39 weeks of data collection. During the execution of the activities, more collaborative teamwork was noticed, because all teams were already aware of their commitments and milestones defined during the schedule meetings phase, thus reducing the transfer of responsibilities on site. In this case study, the phase schedule board with the activities was improved by highlighting the building stories, which provided a more effective visualization of the sequence and parallelism of the activities. As in the Study A, the joint analysis of the metrics selected contributed to the analysis of the adherence between planning levels. Figure 3 presents the results of CL and PCR indicators and PRCO and PPC indicators. The control cycle was 30 days. The phases analyzed were Structure (S), Masonry, Electrical and Plumbing Installation (M/EP), Internal Mortar 
Plaster (IMP), Levelling Mortar (LM), Facade (FA), Drywall System (DS), and Internal Painting (IP).
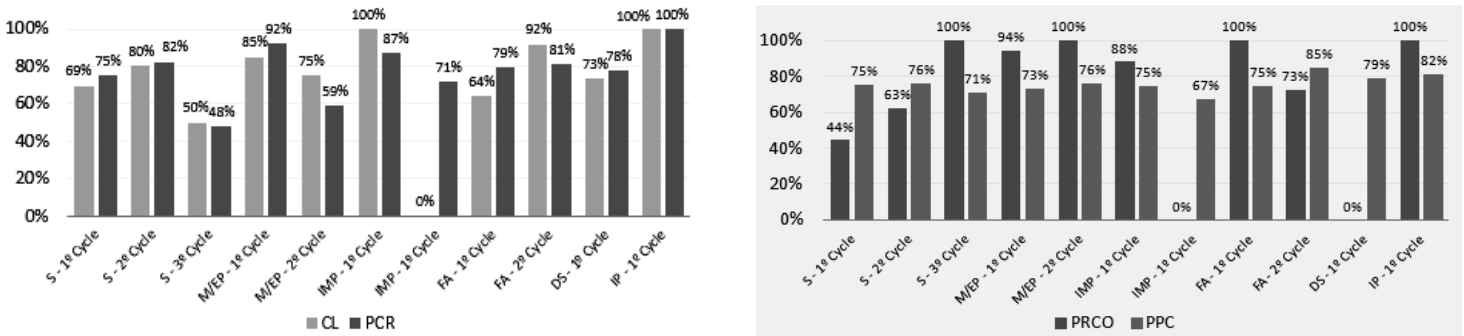

Figure 3: Indicators CL and PCR and PRCO and PPC results of the Case Study B

The team made a greater effort to remove the constraints at the due dates (PCR average $78 \%$ ) and to make the activities ready to be executed (CL average $72 \%$ ), probably because of the learning effect. Although the performance of the PRCO (average of $69 \%$ ) was below the CL indicator, it was noted that the team made an effort to accomplish the second commitment established in Phase Schedule/lookahead planning and to finish the majority of the activities on the scheduled dates, as can be seen in the PRCO results in the Figure 3.

Although the number of Phase Schedule cycles was larger than in the Case Study A, the UAI indicator performance was very high (average 70\%), meaning a high number of activities not planned in Phase Schedule/lookahead planning were performed in the commitment planning, which had a strong influence on the PPC result. In general, the unfinished and unplanned activities in Phase Schedule/lookahead planning contributed to a decrease in the PPC performance, demonstrated by the UUA performance (average of 76\%). It is important to highlight that the result of CL and PRCO indicators of the first levelling mortar cycle were $0 \%$, because the subcontractor responsible for the activities was unable to make the activities ready to start on the planned dates, influencing the finalexecution date. Also, due to the lack of drawings definition, the milestones of the master schedule were delayed.

\section{EFFECTIVENESS OF THE PRACTICES AND PROCESS OF PHASE SCHEDULE}

The effectiveness of the Phase Schedule practices was analyzed based on the constructs defined previously related to collaboration and transparency, and the effectiveness of the processes was analyzed based on the adherence between planning levels, reliability of plans and commitment with the deadlines.

- Collaboration: some strengths related to the collaboration construct were identified, such as: plan commitment, joint decision making and shared knowledge throughout the stages of Phase Schedule cycle; previous discussions between the team involved in setting goals; self-reorganization and better interaction between the team during the execution of the activities in the field; and verification of possible interferences and joint identification of constraints in a collaborative way. 
- Transparency: the strengths identified were: the use of visual tools in the Phase Schedule meetings, allowing the verification of activities that would be performed simultaneously; the simplification of the information to transfer for those involved; and a better visualization of all activities in the phase schedule, facilitating the reorganization and increasing communication on site.

- Adherence between planning levels: by analyzing the CL and PCR indicators, a relation between Phase Schedule and lookahead planning was noted, and it was possible to evaluate whether what was planned concerning the start dates of the activities and the due date for removal of the constraints was in fact achieved. The use of those metrics does not show a statistical correlation, since the PCR indicator only verifies the removal of all constraints at the due dates, not associating those constraints with the activities planned. This means that an activity may have several constraints eliminated, causing a high PCR, while another activity may have only one constraint not eliminated, resulting in a low $\mathrm{CL}$ performance. Analyzing the $\mathrm{CL}$ and PRCO results, there is an adherence between the lookahead planning and the commitment planning. Those provide information as to whether the activities that were ready to be executed during the lookahead were actually planned in the commitment plan and executed within the duration stipulated in the Phase Schedule. According to the results, there is no direct correlation between the CL and PRCO indicators, since other factors may influence the execution of the activities, having an impact on the PRCO results. Analyzing the PRCO and PPC, there is a relation between Phase Schedule and commitment planning, because the activities taken into account in the PRCO are part of the activities analyzed in the PPC, so if there is a high/low PRCO, the PPC will also be influenced by this result. The unplanned activities in Phase Schedule have an impact on the effectiveness of the planning, because there is a high percentage of those activities in the commitment plans that were not concluded. The UAI and UUA indicators complement the analysis of the PRCO and PPC indicators, because both provide information about the activities not planned in the Phase Schedule cycles.

- Reliability of plans: the team that participated in the Phase Schedule meetings was also the one that monitored the constraints and activities planned, contributing to the reliability of plans. This construct can be also analyzed through the commitment to remove the constraints, which can be verified through CL and PCR indicators. For example, if an activity has all its constraints removed on thescheduled date, the activity will be executed as planned, improving the reliability of plans.

- Commitment to the deadlines: the analysis of the complete activities on the expected dates in the master plan was performed through the analysis of the Time Deviation (TD)metric. Throughout the study, two important commitments were identified during the Phase Schedule cycle: (1) starting the activity on the planned date, which was evaluated by the CL, and (2) ending the activity on the planned date, which was evaluated by the PRCO. 


\section{CONCLUSIONS AND FUTURE RESEARCH}

This paper aimed to present the results of the implementation and evaluation of the LPS focusing on the Phase Schedule practices based on two in-depth case studies carried out in Salvador-Brazil.

This paper contributes to a better understanding of the Phase Schedule cycles, involving three main steps: Phase Schedule preparation, Phase Schedule meetings and monitoring of the planned activities and constraints. Also, this study established a set of constructs and metrics for the evaluation of the LPS, focusing on the Phase Schedule practices and processes. The constructs adopted were collaboration, transparency, adherence between planning levels, reliability of plans and commitment to the deadlines, and the metrics selected were Time Deviation (TD), Commitment Level (CL), Percentage of Constraint Removal (PCR), Percent Required Completed or Ongoing (PRCO), Percent Planned Complete (PPC), Unplanned Activities Included in the commitment planning (UAI) and Unplanned and Unfinished Activities in the commitment planning (UUA).

The results show that the constraints analysis, the collaboration between those involved and the transparency in the planning processes were improved in both Case Studies. Also, the metrics selected improved the adherence between planning levels, reliability of plans and commitment to the deadlines. Further, the findings show two main commitments made between those involved in the Phase Schedule cycle:to make the activities ready to be executed and to complete the activity within the deadline planned. The results show the activities which were not analyzed as critical for planning in the Phase Schedule had strong influence on the performance of the PPC.There are still opportunities to analyze the role of new metrics identified in the literature to evaluate the LPS, focusing on the Phase Schedule practices.

\section{REFERENCES}

Ballard, G. (2000). The Last Planner System of Production Control. PhD Diss., University of Birmingham, UK.

Ballard, G. \& Tommelein, I. (2016). Current Process Benchmark for the Last Planner System. Available at: p2sl.berkeley.edu [Accessed 07 Feb. 2018].

Choo, H. J. \& Tommelein, I. D. (2000). WorkMovePlan: Database for Distributed Planning and Coordination. 8th Ann. Conf. of the Int'l Group for Lean Construction.Brighton, UK. <http://www.iglc.net/> (Feb 7, 2018).

Costa, D. B.; Formoso, C. T.; Lima, H. M. R.; Barth, K. B. (2005) Sistemas de Indicadores para Benchmarking na Construção Civil: Manual de Utilização. Porto Alegre, RS: UFRGS/PPGEC/NORIE, 2005. [In Portuguese].

Dave, B., Hämäläinen, J.P., Kemmer, S., Koskela, L., and Koskenvesa, A. (2015). Suggestions to improve lean construction planning. 23rd Ann. Conf. of the Int'l Group for Lean Construction. Perth, Australia. <http://www.iglc.net/>(Feb 7, 2018).

Emdanat, S. and Azambuja, M. (2016). Aligning Near and Long Term Planning for LPS Implementations: A Review of Existing and New Metrics. 24th Ann. Conf. of the Int'l Group for Lean Construction. Boston, USA. <http://www.iglc.net/>(Feb 7, 2018). 
Hamzeh, F.; Ballard, G.; Tommelein, I. D. (2012). "Rethinking Lookahead Planning to Optimize Construction Workflow." Lean Construction Journal, 15-34.

Jang, J. W., Kim, Y. W. (2007). Use of Percent of Constraint Removal to Measure the Make Ready Process. 15th Ann. Conf. of the Int'l Group for Lean Construction. Michigan, USA. <http://www.iglc.net/> (Feb 7, 2018).

Kalsaas, B. T., Skaar, J.,Thorstensen, R. T. (2009). Implementation of Last Planner in a Medium-Sized Construction Site. 17th Ann. Conf. of the Int'l Group for Lean Construction. Taipei, Taiwan. <http://www.iglc.net/>(Feb 7, 2018).

Knapp, S., Charron, R., and Howell, G. (2006). Phase Planning Today. 14th Ann. Conf. of the Int'l Group for Lean Const..Santiago, Chile. <http://www.iglc.net/> (Feb 7, 2018).

Ribeiro, F. S. (2018). "Diretrizes para implementação e avaliação do Sistema Last Planner com foco nas práticas do Planejamento de Fase." Master Diss., Post Graduation Program in Civil Eng., Federal Univ. of Bahia, Salvador, Bahia, Brazil. [In Portuguese]. 